\title{
Clinical characterisation and the improved molecular diagnosis of autosomal dominant cone-rod dystrophy in patients with SCA7
}

\author{
Xuan Zou \\ Peking Union Medical College Hospital \\ Fengxia Yao \\ Peking Union Medical College Hospital \\ Fengrong Li \\ Beijing hospital of traditional Chinese medicine \\ Shijing Wu \\ Peking Union Medical College Hospital \\ Hui Li \\ Peking Union Medical College Hospital \\ Zixi Sun \\ Peking Union Medical College Hospital \\ Tian Zhu \\ Peking Union Medical College Hospital \\ Xing Wei \\ Peking Union Medical College Hospital \\ Donghui Li \\ Peking Union Medical College Hospital \\ Ruifang Sui ( $\square$ hrfsui@163.com) \\ https://orcid.org/0000-0002-6659-0926
}

Research

Keywords:

Posted Date: June 12th, 2020

DOl: https://doi.org/10.21203/rs.3.rs-34275/v1

License: (a) (1) This work is licensed under a Creative Commons Attribution 4.0 International License. Read Full License 


\section{Abstract \\ Purpose}

To evaluate the retinal phenotype and genetic features of Chinese patients with spinocerebellar ataxia type 7 (SCA7).

\section{Methods}

Detailed ophthalmic examinations, including electroretinograms, fundus photography, fundus autofluorescence and optical coherence tomography, were performed to analyse the retinal lesions of patients with SCA7. A molecular genetic analysis was completed to confirm the number of CAG repeats in ATXN7 gene on the patients and their family members.

\section{Results}

Eight patients from three families with SCA7 were included in this study. Trinucleotide repeat was expanded from 43 to 113 in the affected patients. The affected patients were characterised by different degrees of cone-rod dystrophy, which is positively related to the number of CAG repeats and age. All patients complained of progressive bilateral visual loss, and most cases reported visual disturbance earlier than gait movement or dysarthria. A coarse granular appearance of the macular region on scanning laser ophthalmoscopy, hypofluorescence in the macula on autofluorescence, retinal atrophy on optic coherence tomography, depression of multifocal electroretinograms and prominent abnormalities in cone-mediated responses on electrograms are the general features of SCA7-related retinopathy. Hyperreflective dots in the outer retinal layers and choroidal vessel layers are a common sign in optic coherence tomography in the advanced stage.

\section{Conclusions}

SCA shows a cone-rod dystrophy phenotype. The multimodal imaging of the retina is beneficial to detect the early lesions of cone-rod dystrophy related to SCA7.

\section{Introduction}

Spinocerebellar ataxias (SCAs) are a genetically diverse group of autosomal dominant disorders primarily characterised by the degeneration of the cerebellum, brainstem and spinal cord ${ }^{1,2}$. Although these disorders have different clinical features and disease-causing genes, they all share a common underlying mutational mechanism: an expanded CAG repeat encoding a tract of glutamine amino acids (polyglutamine or polyQ tract). Visual system involvement is one of the clinical features of SCAs, and it may be the presenting sign for some types of SCAs. Specifically, the ocular motor features of SCAs include impaired saccadic velocity, impaired smooth pursuit gain, deficits in the vestibulo-ocular reflex and nystagmus. Optic nerve atrophy and retinal degeneration have also been described in certain SCA types ${ }^{3-5}$.

Spinocerebellar ataxia type 7 (SCA7) (OMIM:164500) is caused by the expansion of a CAG triplet repeat that is translated into a polyglutamine tract in ataxin7 (ATXN7). SCA7 is clinically distinguished from other types of SCAs by the additional presence of retinal degeneration. The ophthalmic features of SCA7 are progressive visual disturbance mainly due to retinal degeneration and impaired ocular motility ${ }^{6}$, which produces a very specific diagnosis. Presenting visual symptoms may include photophobia, dyschromatopsia, decreased vision and hemeralopia ${ }^{7}$. The appearance of the fundus in SCA7 is variable, and the reported phenotypes range from normal appearance, occult macular dystrophy (OMD), macular dystrophy and cone-rod dystrophy ${ }^{1,8-11}$. Ocular motor abnormalities may include slowed saccades, saccadic pursuits, saccadic dysmetria and gaze-evoked nystagmus ${ }^{7}$, which are relatively non-specific for olivopontocerebellar atrophies. Decreased corneal endothelial cell density and increased corneal thickness have also been reported ${ }^{2}$.

There have been a number of reports on the visual disturbances in AXTN7-associated retinopathy ${ }^{1,8-11}$. However, there is limited data on the ophthalmic clinical spectrum and genetics of SCA7 from the Chinese population. To complement and extend these investigations, we characterised the retinal function and structure in detail for patients from three families with SCA7.

\section{Patients And Methods}

Eight patients from three families with SCA7 were included in this study (Figure 1). All participants were identified in the Ophthalmic Genetics Clinic at Peking Union Medical College Hospital (PUMCH), Beijing, China. Family members were identified as affected by family history and/or genetic testing. Written informed consent was obtained from either the participating individuals or their guardians. This study was approved by the Institutional Review Board of PUMCH (No. JS-2059) and adhered to the tenets of the Declaration of Helsinki and the Guidance on Sample Collection of Human Genetic Diseases by the Ministry of Public Health of China.

\section{Clinical Evaluation}

The full medical and family history of each participant was obtained. The clinical evaluation included the measurements of the best-corrected decimal visual acuity (BCVA), ophthalmoscopy, fundus photography (Topcon, Tokyo, Japan or HFC, Micro Clear Medical, Suzhou, China), imaging of fundus autofluorescence (FAF) and 633-nm laser of a scanning laser ophthalmoscope (SLO) (Heidelberg Engineering, Germany), optical coherence tomography (OCT) 
(Topcon, Tokyo, Japan or Heidelberg Engineering, Germany) and visual field (VF) testing (Haag-Streit, Koeniz, Switzerland). Electroretinogram (ERG) was recorded according to the standards of the International Society for Clinical Electrophysiology of Vision (RetiPort ERG System, Roland Consult, Wiesbaden, Germany or The RETeval device, LKC, USA) ${ }^{12}$. Multifocal ERGs (VERIS, EDI Inc., USA) were also recorded when possible.

\section{Molecular analyses}

Genomic DNA was isolated from peripheral leukocytes using a QIAamp DNA Blood Midi Kit (Qiagen, Hilden, Germany) according to the manufacturer's protocol. A locus-specific FAM fluorescently labelled forward primer (5'-FAM-TGTTACATTGTAGGAGCGGAA-3') was designed, and the reverse primer was the no-labelled sequence (5'-CACGACTGTCCCAGCATCACTT-3'). Polymerase chain reaction (PCR) was performed in $25 \mu$ reaction volumes containing $20-100$ ng genomic DNA, $10 \mu \mathrm{M}$ of each primer, $2 \times$ KAPA HiFi HotStart PCR Kit and $\mathrm{ddH}_{2} \mathrm{O}$. Initial denaturation was at $95^{\circ} \mathrm{C}$ for 5 min, followed by amplification for 35 cycles with denaturation at $95^{\circ} \mathrm{C}$ for $45 \mathrm{~s}$, annealing at $55^{\circ} \mathrm{C}$ for $50 \mathrm{~s}$ and extension at $72{ }^{\circ} \mathrm{C}$ for $60 \mathrm{~s}$. A total of $1 \mu$ of the reaction products was added to 12 $\mu \mathrm{l}$ of $\mathrm{Hi}$-Di formamide and $0.5 \mu \mathrm{l} \mathrm{GeneScan} 500 \mathrm{LIZ}$ Size Standard, denatured at $95^{\circ} \mathrm{C}$ for $2 \mathrm{~min}$ and immediately placed on ice for a minimum of $3 \mathrm{~min}$. The samples were injected in an ABI3500xl with a $50 \mathrm{~cm}$ capillary containing POP7. The amplicon length was calculated in comparison with the GeneScan 500 LIZ by the GeneMarker V1.5 demo program.

To precisely assess the number of CAG repeats, one normal control with two homozygous controls was Sanger-sequenced. Sanger sequencing was performed using the BigDye Terminator Cycle Sequencing Ready Reaction Kit version 3.1 (Applied Biosystems, Thermo Fisher Scientific, Inc., Waltham, MA, USA), and the sequence was analysed with an $A B I$ Prism 3130 automated sequencer. The sequencing results were compared against the reference genomic sequence obtained from the University of California, Santa Cruz (Santa Cruz, CA, USA) Genome Browser (https://genome.ucsc.edu/; ATXN7, NM_000333). Twenty-five healthy controls were tested to calculate the Chinese allele frequencies in the population.

To determine the exact number of CAG repeats for each tested sample and obtain the standard profile of a homozygous individual, one homozygous genotype was selected. The normal homozygous control by Sanger sequence and capillary electrophoresis fragment analysis contained 10 CAG repeats, and the correspondence length was $294 \mathrm{bp}$. Thus, the number of CAG repeats was [the length -(294-30)]/3.

\section{Case reports}

\section{Family 1 Case III:3}

A 54-year-old woman noticed decreased visual acuity and mild photophobia at age 45. Gait disturbance and dysarthria occurred at age 51 . Brain magnetic resonance imaging (MRI) demonstrated mild cerebellar atrophy. When she consulted us, her BCVA was 0.1 OU with a refraction of $+1.50 \times 60$ OD and $+1.0 \times$ 100 OS. Intraocular pressure was $11 \mathrm{mmHg}$ in both eyes. Nystagmus was not detected. Slit-lamp biomicroscopic examination showed that the anterior segments were unremarkable. The fundus photography demonstrated a coarse granular depigmentation in the macular area (Figures $2 \mathrm{~A}, 2 \mathrm{~B}$ ), which corresponded with a round hypofluorescence patch with a surrounding hyperfluorescent ring on FAF (Figures 3A, 3B). OCT revealed a disorganised retinal structure in the macula, thinning of the retinal pigment epithelium (RPE) and absence of an ellipsoid zone and an outer limiting membrane. Multiple hyperreflective dots above and beneath the retinal RPE layer were detected. A mild epiretinal membrane formed in the left eye (Figures $4 A-4 D)$. A coarse granular appearance of the macula was observed in SLO (Figures 5A, 5B). VF analysis showed a central scotoma in both eyes. The ERGs exhibited severely decreased cone responses and a relatively normal rod function (Figure 6). Multifocal ERGs demonstrated a depression of the response amplitude in the macular area. The CAG repeat sequences in ATXN7 were extended to 45.

\section{Family 1 Case IV:5}

A 32-year-old man, the nephew of Case III:3, complained of blurred vison and photophobia at age 26 and a gait disturbance and dysarthria at age 30 . Computed tomographic scans and magnetic resonance images showed atrophy in the cerebellar region. His BCVA was 0.4 OD and 0.32 OS. His eye movements were full without nystagmus. Slit-lamp biomicroscopic examination revealed that the anterior segment was normal in both eyes. The fundus showed macular atrophy with surrounding pigment changes (Figures 2C, 2D) similar to those of Case III:3. The ERGs demonstrated severely decreased cone responses and a preserved rod function. The number of CAG repeats in ATXN7 was 50.

\section{Other affected members from Family 1}

Two other family members were affected, and they were confirmed by genetic testing without ophthalmic examination. Case III:9, a 57-year-old man, noticed gait disturbance, dysarthria and decreased visual acuity in his 40 s. He was wheelchair-bound, and vision was hand motion in both eyes, and was deceased at the age 58. The CAG repeat sequences in ATXN7 were extended to 43. Case IV:4, a 26-year-old man, had blurred vision in his teenage years and gait disturbance and dysarthria at age 18. He had been paralysed and had no light perception in both eyes for about six years. He had the most severe symptom in Family 1. The number of expanded CAG repeats was 56.

\section{Family 2}

A 14-year-old girl (Case II:1) presented with decreased visual acuity at age 12 and gait disturbance at age 13. Brain MRI revealed a mild volume loss in the cerebellum. Her BCVA was 0.1 OD with a refraction of $-1.0-1.0 \times 3$ and 0.08 OS with a refraction of -1.75 at the first visit. The anterior segments were normal. The fundus showed about two disc-sized atrophic lesions in a petal-like shape with surrounding pigment changes (Figures 2E, 2F), and the FAF manifested a hypofluorescent macula and mottled fluorescence in the posterior pole (Figures 3C, 3D). OCT demonstrated a preserved laminar retinal structure in the macula, a thinning of the RPE and the absence of an ellipsoid zone and an outer limiting membrane, and there were a few hyperreflective dots in the outer layers of the retina (Figures 4E-4H). VF analysis indicated a central scotoma in both eyes. The ERGs revealed severely decreased cone responses with a limited preserved 
rod function. The patient's ERGs became non-recordable(Figure 6), and BCVA decreased to 0.04 (OD) and 0.03 (OS) three years later during a follow-up visit. The CAG repeat sequence in ATXN7 was extended to 62.

Her father (l:1) did not have a vision complaint and showed a normal OCT structure (Figures $4 \mathrm{I}, 4 \mathrm{~J}$ ) when he first visited us at age 38 to accompany her daughter. His ERGs were normal both in the dark-adapted and light-adapted responses(Figure 6). One year later, he began to notice a gradually decreasing visual acuity. He was genetically confirmed by the expanded CAG repeats of 45 . He consulted us again at age 42 with BCVA of 0.1 OU with a refraction of $-0.75 \times 80 \mathrm{OD}$ and $-0.5-1.0 \times 85 \mathrm{OS}$. No specific abnormality was found on fundus examination (Figures $2 \mathrm{G}, 2 \mathrm{H}$ ). However, OCT showed a mild thinning of the macula and atrophic changes in the RPE and the ellipsoid zone. A reduction of central responses was demonstrated in multifocal ERGs.

\section{Family 3}

A 32-year-old man (Case III:2) reported decreased visual acuity, hemeralopia and photophobia at age 28 and gait disturbance at age 30 . Computed tomographic scans and magnetic resonance images showed cerebellar atrophy. His BCVA was 0.1 OU with a refraction of $-0.5-0.5 \times 15$ OD and -0.5 OS. The anterior segment examination was normal. The fundus revealed pigmentary changes in the macular area (Figures $2 \mathrm{I}, 2 \mathrm{~J}$ ), which corresponded with the AF hypofluorescence patch in a rounded square shape with a surrounding hyperfluorescent ring (Figures 3E, 3F). OCT manifested macular atrophy, extreme thinning of the fovea and many hyperreflective dots (Figures $4 \mathrm{~K}-4 \mathrm{~N}$ ). VF analysis confirmed a central scotoma in both eyes. The ERGs presented severely decreased cone-rod responses(Figure 6). The pathological allele carried 48 CAG repeats. His son (IV:1) was a four-year-old boy who began to be unable to walk and pursue objects at age 2 . He was bed ridden with respiratory failure and lost light perception at age 4 . He was confirmed by gene testing, with CAG repeats of 113 .

The results of the clinical characterisation and genetic test of all patients are summarised in Table 1 . The CAG repeat numbers of the 30 normal controls was $(\mathrm{CAG})_{7}(6 / 60,10.0 \%),(\mathrm{CAG})_{10}(41 / 60,68.3 \%),(\mathrm{CAG})_{11}(2 / 60,3.3 \%)$ and $(\mathrm{CAG})_{12}(11 / 60,18.3 \%)$.

\section{Discussion}

SCA7 is a form of SCA with a proportion of $2 \%-26.6 \%$ in different regions worldwide ${ }^{13-18}$, with a prevalence of around $1 / 500,000^{19}$. SCA7 is the most common dominant ataxia in Sweden, Norway, Denmark and Finland as a result of the founder effect ${ }^{17}$. In the Chinese population, SCA3 has been reported as the most common subtype of SCAs (54.6\%), and SCA7 accounts for only $4.8 \%$ of SCAs ${ }^{18}$. In SCA7 patients, the unstable expanded trinucleotide repeats of CAG induce a cytotoxic gain of function of ataxin-7. It localises to the nucleus and/or the cytoplasm depending on the cell population and is widely expressed in human tissues ${ }^{20,21}$. In the retina, ataxin-7 is present in all neurons located in nuclei and inner segments of photoreceptors and absent in their outer segments ${ }^{2}$. The expression of polyQ-ataxin-7 in AXTN7 has been known to cause the cellular loss of specific neuronal cells, including retinal photoreceptors, through a toxic effect that causes a downregulation of the genes involved in photo-transduction, development and differentiation of photoreceptors ${ }^{22}$.

The size of the pathogenic CAG repeats in ATXN7 is inversely correlated with the age of onset of symptoms and the rate of disease progression ${ }^{1,2,19,23}$. SCA7 patients have been reported to have 37-460 CAG repeats compared with the 4-35 CAG repeats in the normal allele ${ }^{1,18,24,25}$, with the earliest onset during infancy ${ }^{26}$. The number of pathological CAG repeats in our patients was $43-113$, and the repeat number 10 of CAG was the most common in the Chinese population. In our cohort, for patients with CAG repeats of $43-45$, the onset age was in their $40 \mathrm{~s}$; for patients with $48-50$ repeats, the onset age was in their 20s; and for patients with 56-62repeats, symptoms began at teenage years. If the CAG repeats reached more than 100, symptoms would begin at childhood and worsen rapidly, thus making them life threatening. With relatively lower CAG repeats, patients could be asymptomatic. Similar to our case Il: 1 from Family 2 (CAG repeats $=45$ ), he had no complaints when he was 38 , and clinical examination revealed no obvious abnormality in the retina. During his four-year follow-up, we noticed a gradually progressing cone dysfunction, which did not affect his normal life. Jun Young Park et al. ${ }^{10}$ reported a case of a 52 -year-old woman who presented with a lower number (38) of CAG repeats in ATXN7 and a mild form of retinal degeneration, which was similar to the classic type of OMD. However, in this case, the follow-up period lasted only eight months. It is possible that this case would develop to cone-rod dystrophy over the next few years. OMD is a certain stage for these patients with a lower number of CAG repeats. Conversely, the phenotype in patients with CAG repeats of more than 100 could be very severe. For example, our youngest patient, case IV:1 from Family 3, with 113 CAG repeats exhibited severe vision loss and dyskinesia, and he deteriorated rapidly within two years. Our findings are consistent with those in the literature in that long expansions associated with infantile-onset SCA7 occurred exclusively in paternal transmission ${ }^{11}$.

Clinically, visual disturbances may precede other symptoms in SCA7, and multimodal imaging may facilitate the observation of retinal change and is beneficial to detect early lesions of $A T X N 7$-related retinopathy. The retinal phenotype in our patients indicating a cone-rod dystrophy generally agrees with previous reports ${ }^{1,8-11}$. In our cohort, all the patients complained of progressive bilateral visual loss, and most cases reported visual disturbance earlier than dyskinesia or dysarthria. The severity of retinopathy can vary from a normal retina appearance to severe cone-rod dystrophy, and it is correlated with the number of CAG repeats and age. The thinning of the macular tissue on OCT and the dark macular area on AF demonstrated the severity and the area of macular dystrophy, including diffuse or local photoreceptor degeneration and disruption of the RPE. The shape of the hypofluorescent area in the macula could be round, petal-like and rounded square. In some patients, we observed a hyperfluorescent ring around a dark macula on AF, indicating the expansion of the macular atrophy. Abnormalities in the ERGs tend to be more prominent in cone-mediated responses or in both cone and rod signals, which are the features of an evolving cone-rod dystrophy depending on the stage of the disease and the number of CAG repeats. We also noticed that in case Il: 1 from Family 2 , the ERGs evolved from residual weak rod responses to extinguished within a four-year follow-up.

We notice a common sign of OCT in our patients in the advanced stage, that is, hyperreflective dots in the outer retinal layers, the number of which decreases in the late stage. We reviewed an OCT scan in a SCA7 patient reported previously and found that the hyperreflective dots could also be easily identified ${ }^{19}$, indicating that this appearance could be an OCT feature for SCA7 patients. The hyperreflective dots seemed to correspond to the coarse granular appearance

Page $4 / 11$ 
on the 633-nm laser SLO, but they were not visible on the fundus photograph or AF. The hyperreflective dots were not seen in patients at the early stage (Family 2-Case II:1), and the number was fewer in patients at the late stage (Family 2-Case I:1), indicating a lesion at a certain stage of the disease. Furthermore, the dots tended to accumulate at the border of a dystrophic and a relatively normal retina. Polyglutamine expansion in ATXN7 causes its misfolding and prominent intranuclear accumulation, disrupting a wide range of cellular processes and finally leading to the death of photoreceptors ${ }^{27}$. The hyperreflective dots were likely to be the degenerative photoreceptors and reflected the phagocytosis of photoreceptor outer segments. The dense hyperreflective dots in the choroidal vessel layers are also observed in other retinal diseases, such as Stargardt's disease ${ }^{28}$ and central serous chorioretinopathy ${ }^{29}$. The present study clearly demonstrates that the hyperreflective dots are more prevalent in the choroidal layers, specifically in the Bruch membrane/RPE complex and choriocapillaris. The reason for the presence of hyperreflective foci in the choroid is uncertain. As the cause of the retinal and neuronal dysfunction and how it is related to the polyglutamine repeat is not yet fully understood.

In this study, we also improved the molecular diagnostic method for testing patients with SCA7. We used the simultaneous Sanger sequencing for homozygous individuals to calibrate the CAG expansions of the fluorescence PCR. Fluorescence PCR and capillary electrophoresis for the detection of dynamic mutations, such as ATXN7, have the advantages of being simple, fast and cost effective. However, this method should be used to guard against false negative results, and it needs to cooperate with other methods, such as Southern blot.

In summary, we provided a detailed retinal study on patients with SCA7, which could be considered cone-rod dystrophy. We also noted an important feature, that is, hyperreflective dots, on OCT and improved the molecular diagnostic method of SCA7. Our case series illustrates that multimodal imaging of the retina is beneficial to detect early lesions of cone-rod dystrophy related to SCA7 and that this type of imaging could help ophthalmologists produce a specific diagnosis.

\section{Declarations}

\section{Ethics approval and consent to participate}

This study was approved by the Institutional Review Board of PUMCH (No. JS-2059) and adhered to the tenets of the Declaration of Helsinki and the Guidance on Sample Collection of Human Genetic Diseases by the Ministry of Public Health of China.

\section{Consent for publication}

Written informed consent was obtained from either the participating individuals or their guardians.

\section{Availability of data and material}

The datasets used and/or analysed during the current study are available from the corresponding author on reasonable request.

\section{Competing interests}

The authors declare that they have no competing interests.

\section{Funding}

This study was supported by the Fundamental Research Funds for the Central Universities (3332020015), Youth Program of Peking Union Medical College Hospital (pumch201911263), National Natural Science Foundation of China (81873687) and CAMS Innovation Fund for Medical Sciences of China (CIFMS 2016-12M-1-002).

\section{Authors' contributions}

XZ wrote the main manuscript text and collected two pedegrees. FXY performed genetic testing and wrote part of the manuscript. SJW, TZ, ZXS, HL and XW collected the patient data and extracted the DNA. DHL performed OCT and FAF examinations. FRL collected one patient. RFS conceived the study.

\section{Acknowledgements}

We are grateful to all the patients and their family members for their participation in this study.

\section{List Of Abbreviations}

SCAs:Spinocerebellar ataxias; SCA7: Spinocerebellar ataxia type 7; ATXN7: ataxin-7; OMD: occult macular dystrophy; BCVA: best-corrected decimal visual acuity; FAF: fundus autofluorescence; SLO: scanning laser ophthalmoscope; OCT: optical coherence tomography; VF: visual field; ERG: Electroretinogram; MRI: magnetic resonance imaging; RPE:retinal pigment epithelium.

\section{References}

1. Atadzhanov M, Smith DC, Mwaba MH, Siddiqi OK, Bryer A, Greenberg LJ. Clinical and genetic analysis of spinocerebellar ataxia type 7 (SCA7) in Zambian families. Cerebellum Ataxias 2017;4:17.

2. Niewiadomska-Cimicka A, Trottier Y. Molecular Targets and Therapeutic Strategies in Spinocerebellar Ataxia Type 7. Neurotherapeutics 2019;16:1074-96. 
3. Jin DK, Oh MR, Song SM, et al. Frequency of spinocerebellar ataxia types 1,2,3,6,7 and dentatorubral pallidoluysian atrophy mutations in Korean patients with spinocerebellar ataxia. J Neurol 1999;246:207-10.

4. Pogacar S, Ambler M, Conklin WJ, O'Neil WA, Lee HY. Dominant spinopontine atrophy. Report of two additional members of family W. Arch Neurol 1978;35:156-62.

5. Jardim LB, Pereira ML, Silveira I, Ferro A, Sequeiros J, Giugliani R. Machado-Joseph disease in South Brazil: clinical and molecular characterization of kindreds. Acta Neurol Scand 2001;104:224-31.

6. Sun YM, Lu C, Wu ZY. Spinocerebellar ataxia: relationship between phenotype and genotype - a review. Clinical genetics 2016;90:305-14.

7. Miller RC, Tewari A, Miller JA, Garbern J, Van Stavern GP. Neuro-ophthalmologic features of spinocerebellar ataxia type 7. J Neuroophthalmol 2009;29:180-6.

8. Abe T, Tsuda T, Yoshida M, et al. Macular degeneration associated with aberrant expansion of trinucleotide repeat of the SCA7 gene in 2 Japanese families. Archives of ophthalmology (Chicago, III : 1960) 2000;118:1415-21.

9. Aleman TS, Cideciyan AV, Volpe NJ, Stevanin G, Brice A, Jacobson SG. Spinocerebellar ataxia type 7 (SCA7) shows a cone-rod dystrophy phenotype. Experimental eye research 2002;74:737-45.

10. Park JY, Wy SY, Joo K, Woo SJ. Spinocerebellar ataxia type 7 with RP1L1-negative occult macular dystrophy as retinal manifestation. Ophthalmic genetics 2019;40:282-5.

11. Michalik A, Martin JJ, Van Broeckhoven C. Spinocerebellar ataxia type 7 associated with pigmentary retinal dystrophy. European journal of human genetics : EJHG 2004;12:2-15.

12. McCulloch DL, Marmor MF, Brigell MG, et al. ISCEV Standard for full-field clinical electroretinography (2015 update). Doc Ophthalmol 2015;130:1-12.

13. Bryer A, Krause A, Bill P, et al. The hereditary adult-onset ataxias in South Africa. Journal of the neurological sciences 2003;216:47-54.

14. Smith DC, Bryer A, Watson LM, Greenberg LJ. Inherited polyglutamine spinocerebellar ataxias in South Africa. South African medical journal = SuidAfrikaanse tydskrif vir geneeskunde 2012;102:683-6.

15. Garcia-Velazquez LE, Canizales-Quinteros S, Romero-Hidalgo S, et al. Founder effect and ancestral origin of the spinocerebellar ataxia type 7 (SCA7) mutation in Mexican families. Neurogenetics 2014;15:13-7.

16. Storey E, du Sart D, Shaw JH, et al. Frequency of spinocerebellar ataxia types 1, 2, 3, 6, and 7 in Australian patients with spinocerebellar ataxia. American journal of medical genetics 2000;95:351-7.

17. Jonasson J, Juvonen V, Sistonen P, et al. Evidence for a common Spinocerebellar ataxia type 7 (SCA7) founder mutation in Scandinavia. European journal of human genetics : EJHG 2000;8:918-22.

18. Wang J, Xu Q, Lei L, et al. [Studies on the CAG repeat expansion in patients with hereditary spinocerebellar ataxia from Chinese Han]. Zhonghua yi xue yi chuan xue za zhi = Zhonghua yixue yichuanxue zazhi = Chinese journal of medical genetics 2009;26:620-5.

19. Niu C, Prakash TP, Kim A, et al. Antisense oligonucleotides targeting mutant Ataxin-7 restore visual function in a mouse model of spinocerebellar ataxia type 7. Science translational medicine 2018;10.

20. Einum DD, Townsend JJ, Ptacek LJ, Fu YH. Ataxin-7 expression analysis in controls and spinocerebellar ataxia type 7 patients. Neurogenetics 2001;3:8390.

21. Lindenberg KS, Yvert G, Muller K, Landwehrmeyer GB. Expression analysis of ataxin-7 mRNA and protein in human brain: evidence for a widespread distribution and focal protein accumulation. Brain pathology (Zurich, Switzerland) 2000;10:385-94.

22. Abou-Sleymane G, Chalmel F, Helmlinger D, et al. Polyglutamine expansion causes neurodegeneration by altering the neuronal differentiation program. Hum Mol Genet 2006;15:691-703.

23. Sequeiros J, Martindale J, Seneca S, et al. EMQN Best Practice Guidelines for molecular genetic testing of SCAs. European journal of human genetics : EJHG 2010;18:1173-6.

24. Durr A. Autosomal dominant cerebellar ataxias: polyglutamine expansions and beyond. The Lancet Neurology 2010;9:885-94.

25. Takahashi T, Katada S, Onodera O. Polyglutamine diseases: where does toxicity come from? what is toxicity? where are we going? Journal of molecular cell biology 2010;2:180-91.

26. Benton CS, de Silva R, Rutledge SL, Bohlega S, Ashizawa T, Zoghbi HY. Molecular and clinical studies in SCA-7 define a broad clinical spectrum and the infantile phenotype. Neurology 1998;51:1081-6.

27. La Spada AR, Taylor JP. Repeat expansion disease: progress and puzzles in disease pathogenesis. Nature reviews Genetics 2010;11:247-58.

28. Piri N, Nesmith BL, Schaal S. Choroidal hyperreflective foci in Stargardt disease shown by spectral-domain optical coherence tomography imaging: correlation with disease severity. JAMA Ophthalmol 2015;133:398-405.

29. Hanumunthadu D, Matet A, Rasheed MA, Goud A, Vuppurabina KK, Chhablani J. Evaluation of choroidal hyperreflective dots in acute and chronic central serous chorioretinopathy. Indian J Ophthalmol 2019;67:1850-4.

\section{Tables}

Table 1 the clinical characterisation and genetic test of patients with SCA7 


\begin{tabular}{|c|c|c|c|c|c|c|c|c|c|c|c|c|}
\hline $\begin{array}{l}\text { Family } \\
\text { No. }\end{array}$ & $\begin{array}{l}\text { Patient } \\
\text { No. }\end{array}$ & $\begin{array}{l}\text { Age } \\
\text { (y) }\end{array}$ & Sex & $\begin{array}{l}\text { Age at } \\
\text { onset } \\
\text { Ophthalmol }\end{array}$ & $\begin{array}{l}\text { Age at } \\
\text { onset } \\
\text { cerebellar }\end{array}$ & $\begin{array}{l}\text { Expanded } \\
\text { Repeat }\end{array}$ & $\begin{array}{l}\text { BCVA } \\
\text { OD }\end{array}$ & $\begin{array}{l}\text { BCVA } \\
\text { OS }\end{array}$ & ОСТ & VF & $\mathrm{AF}$ & ERGs \\
\hline \multirow[t]{4}{*}{1} & $\begin{array}{l}\text { Case } \\
\text { III:3 }\end{array}$ & 54 & $\mathrm{~F}$ & 45 & 51 & $\approx 45$ & 0.1 & 0.1 & $\begin{array}{l}\text { Macular } \\
\text { atrophy with } \\
\text { hyperreflective } \\
\text { dots }\end{array}$ & $\begin{array}{l}\text { Central } \\
\text { scotoma }\end{array}$ & $\begin{array}{l}\text { Dark macular } \\
\text { with } \\
\text { hyperfluorescent } \\
\text { ring }\end{array}$ & $\begin{array}{l}\text { Severel: } \\
\text { decreas } \\
\text { cone } \\
\text { respons } \\
\text { with } \\
\text { preservi } \\
\text { rod funi }\end{array}$ \\
\hline & $\begin{array}{l}\text { Case } \\
\text { III:9 }\end{array}$ & 57 & $M$ & $40 \mathrm{~s}$ & $40 \mathrm{~s}$ & $\approx 43$ & NA & NA & NA & NA & NA & NA \\
\hline & $\begin{array}{l}\text { Case } \\
\text { IV:5 }\end{array}$ & 32 & $M$ & 26 & 30 & $\approx 50$ & 0.4 & 0.32 & NA & NA & NA & $\begin{array}{l}\text { Severel: } \\
\text { decreas } \\
\text { cone } \\
\text { respons } \\
\text { with } \\
\text { preserv } \\
\text { rod funi }\end{array}$ \\
\hline & $\begin{array}{l}\text { Case } \\
\text { IV:4 }\end{array}$ & 26 & $M$ & Teenage & 18 & $\approx 56$ & $\mathrm{HM}$ & $\mathrm{HM}$ & NA & NA & NA & NA \\
\hline \multirow[t]{2}{*}{2} & $\begin{array}{l}\text { Case } \\
\text { Il:1 }\end{array}$ & 14 & $\mathrm{~F}$ & 12 & 13 & $\approx 62$ & 0.1 & 0.08 & $\begin{array}{l}\text { Macular } \\
\text { atrophy with } \\
\text { hyperreflective } \\
\text { dots }\end{array}$ & $\begin{array}{l}\text { Central } \\
\text { scotoma }\end{array}$ & $\begin{array}{l}\text { Dark macular } \\
\text { with } \\
\text { hyperfluorescent } \\
\text { ring }\end{array}$ & $\begin{array}{l}\text { Severel! } \\
\text { decreas } \\
\text { cone } \\
\text { respons } \\
\text { with a li } \\
\text { preservi } \\
\text { rod funı } \\
\text { (age } 14 \\
\text { Extingu } \\
\text { (age 1\& }\end{array}$ \\
\hline & $\begin{array}{l}\text { Case } \\
\mathrm{I}: 1\end{array}$ & 38 & $M$ & 39 & $40 s$ & $\approx 45$ & $\begin{array}{l}0.1 \text { (age } \\
42)\end{array}$ & $\begin{array}{l}0.1 \text { (age } \\
42)\end{array}$ & $\begin{array}{l}\text { Normal(age } \\
38)\end{array}$ & NA & NA & $\begin{array}{l}\text { Normal } \\
38)\end{array}$ \\
\hline \multirow[t]{2}{*}{3} & $\begin{array}{l}\text { Case } \\
\text { III:2 }\end{array}$ & 32 & $M$ & 28 & 30 & $\approx 48$ & 0.1 & 0.1 & $\begin{array}{l}\text { Macular } \\
\text { atrophy with } \\
\text { hyperreflective } \\
\text { dots }\end{array}$ & $\begin{array}{l}\text { Central } \\
\text { scotoma }\end{array}$ & $\begin{array}{l}\text { Dark macular } \\
\text { with } \\
\text { hyperfluorescent } \\
\text { ring }\end{array}$ & $\begin{array}{l}\text { Severel: } \\
\text { decreas } \\
\text { cone an } \\
\text { rod } \\
\text { respons }\end{array}$ \\
\hline & $\begin{array}{l}\text { Case } \\
\text { IV: } 1\end{array}$ & 4 & $M$ & 2 & 2 & $\approx 113$ & NLP & NLP & NA & NA & NA & NA \\
\hline
\end{tabular}

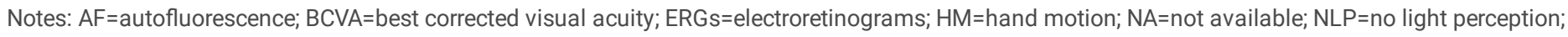
$\mathrm{OCT}=$ optical coherence tomography; $\mathrm{VF}=$ visual field

\section{Figures}

Family 1

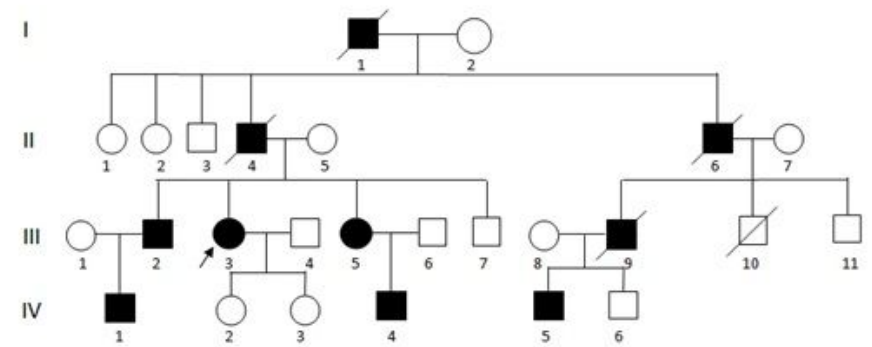

Family 2

Family 3
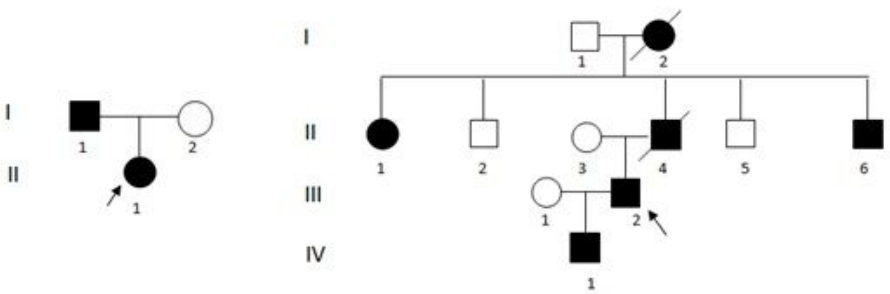

Figure 1 
Pedigrees of the families with variants in AXTN7. The circles represent females, and the squares represent males. The black symbols denote the individuals affected with SCA7. The arrow indicates the proband. The slashed symbols indicate the deceased members.

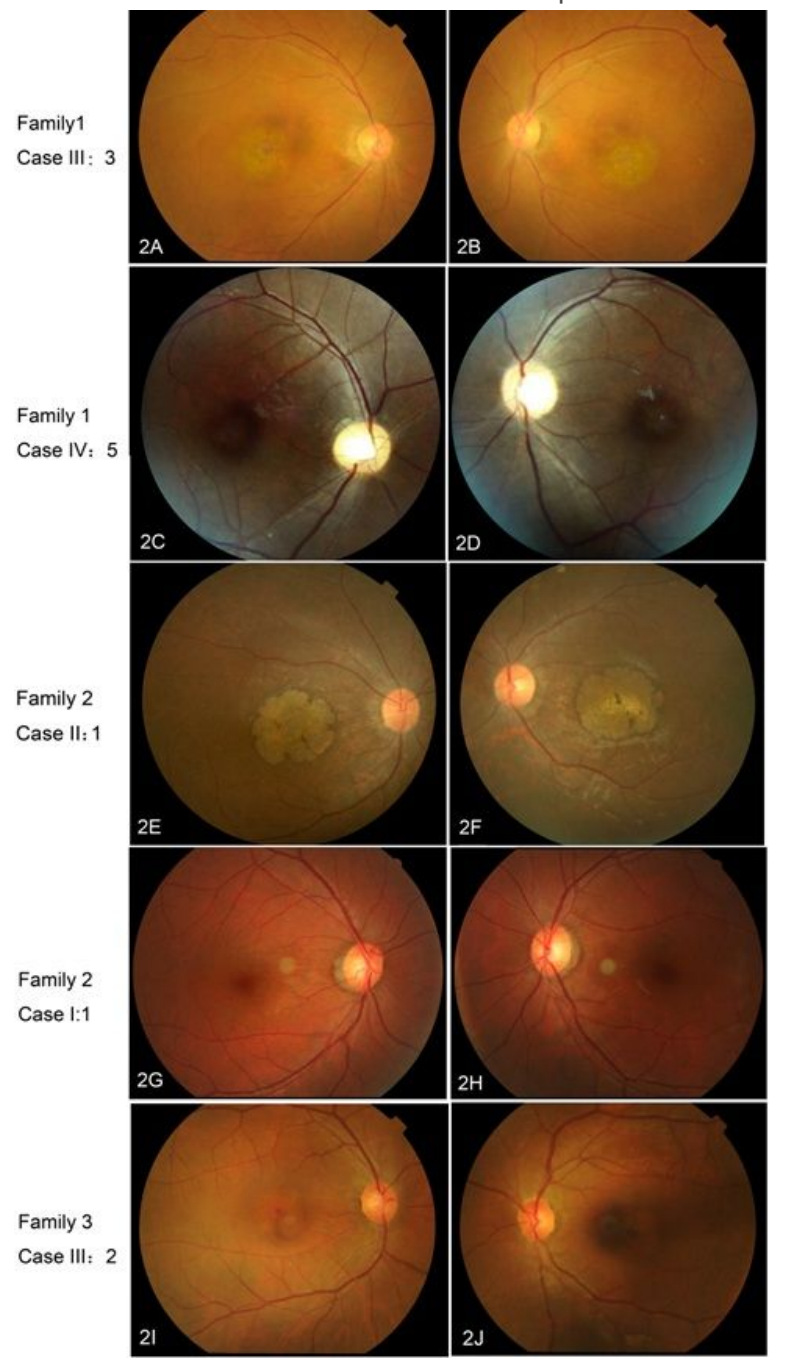

\section{Figure 2}

Fundus photographs of patients with AXTN7 variants. 2A and 2B. Family 1-Case III:3 is a 54-year-old woman. The fundus shows obvious macular atrophy. $2 \mathrm{C}$ and 2D. Family 1-Case IV:5 is a 32-year-old man. The fundus also shows obvious macular atrophy. 2E and 2F. Family 2-Case II:1 is an14-year-old girl. The fundus showed about two disc-sized atrophic lesions in a petal-like shape with surrounding pigment changes. $2 \mathrm{G}$ and $2 \mathrm{H}$. Fundus photographs were taken during the second visit of Family 2-Case l:1 when he was 42 years old. The fundus shows a relatively normal appearance. $2 \mathrm{I}$ and $2 \mathrm{~J}$. Family $3-\mathrm{Case}$ III:2 is a 32-year-old man. The fundus shows obvious macular atrophy. 


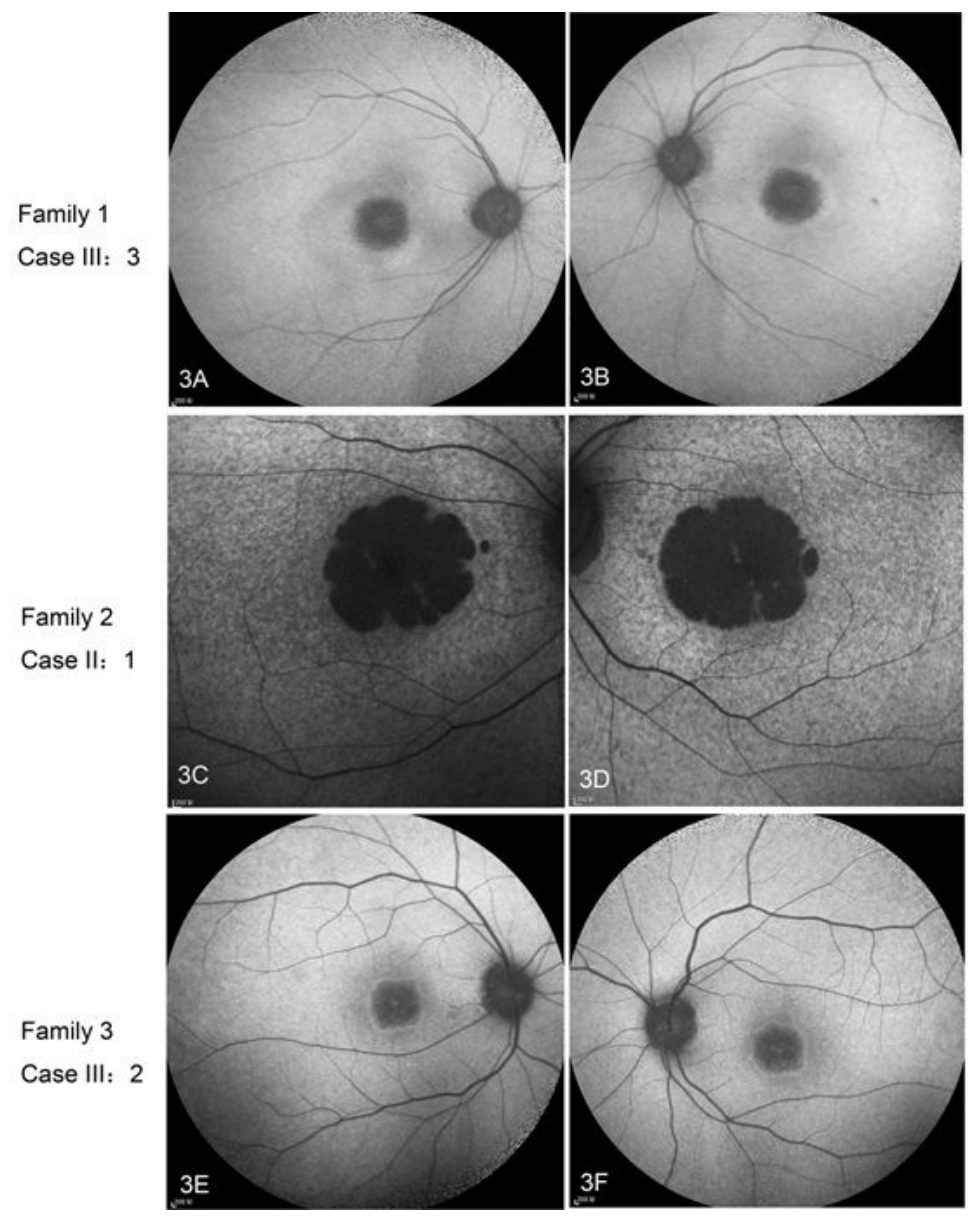

\section{Figure 3}

AF in patients with AXTN7 variants. 3A and 3B. Family 1-Case III:3 is a 54-year-old woman. AF shows a hypofluorescence patch in the macular area with a surrounding hyperfluorescent ring. $3 \mathrm{C}$ and 3D. AF was taken during the second visit of Family 2-Case II:1 when she was 18 years old. AF shows a dark macula and mottled fluorescence in the posterior pole. $3 \mathrm{E}$ and 3F. Family 3-Case III:2 is a 32-year-old man. AF shows a hypofluorescence patch in the macular area with a surrounding hyperfluorescent ring. 


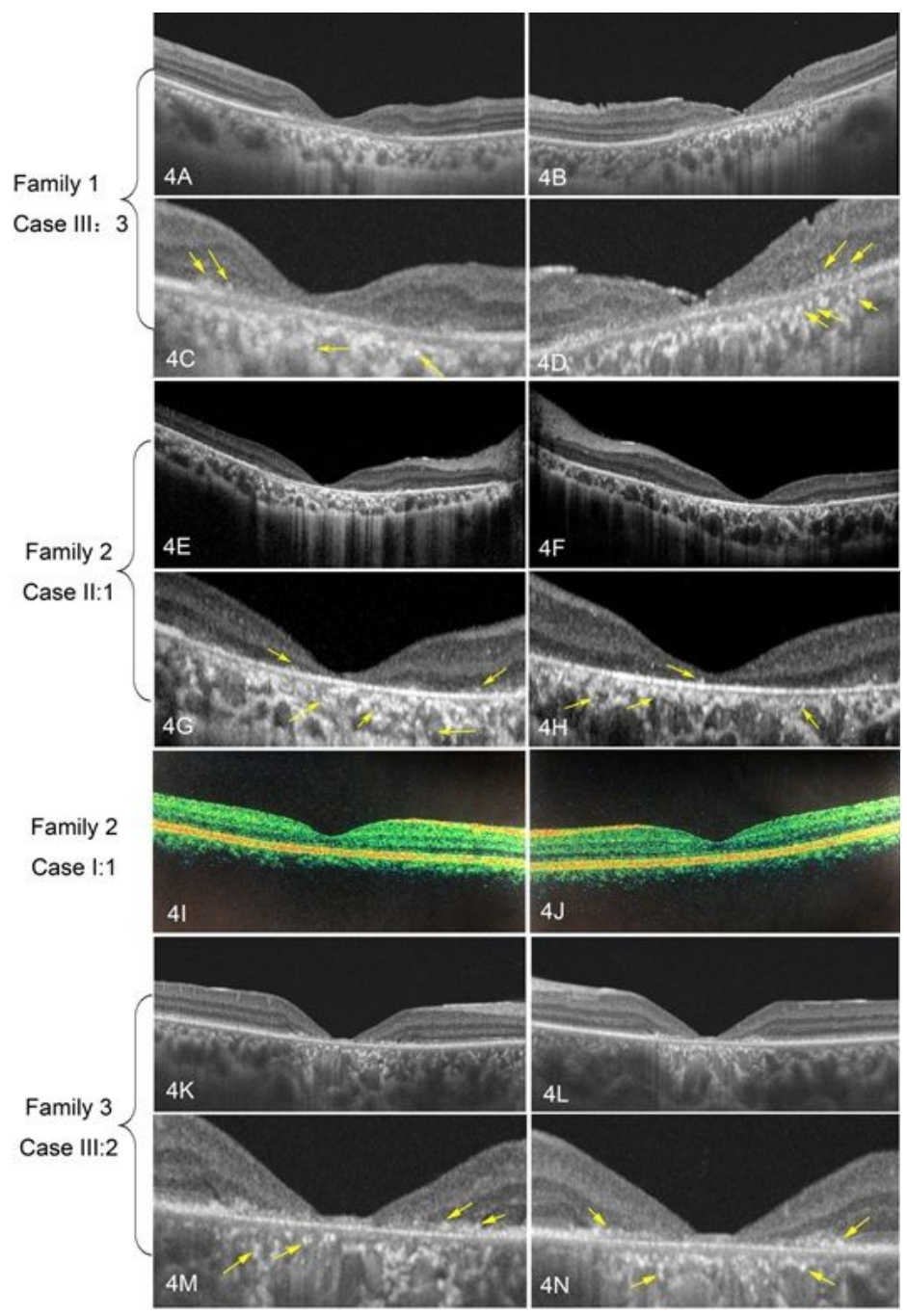

\section{Figure 4}

OCT of patients with AXTN7 variants. Family 1-Case III:3 (a 54-year-old woman) (4A-4D), Family 2-Case II:1 (an 18-year-old girl during the second visit) (4E$4 \mathrm{H}$ ) and Family 3-Case III:2 (a 32-year-old man) (4K-4N) have a similar appearance. OCT shows the thinning of the macula, with the outer layers and RPE being affected more severely. The ellipsoid zone disappears in the fovea area, indicating a CORD appearance. There are many intraretinal hyperreflective dots, which are indicated by the yellow circle. Figures $4 \mathrm{C}$ and $4 \mathrm{D}$ are the enlargement of Figures $4 \mathrm{~A}$ and $4 \mathrm{~B}$, Figures $4 \mathrm{G}$ and $4 \mathrm{H}$ are the enlargement of $4 \mathrm{E}$ and $4 \mathrm{~F}$, and Figures $4 \mathrm{M}$ and $4 \mathrm{~N}$ are the enlargement of $4 \mathrm{~K}$ and $4 \mathrm{~L}$, respectively. Family 2 Case I:I ( $4 \mathrm{I}$ and $4 \mathrm{~J}$ ) showed a normal OCT when he first consulted us at age 38.

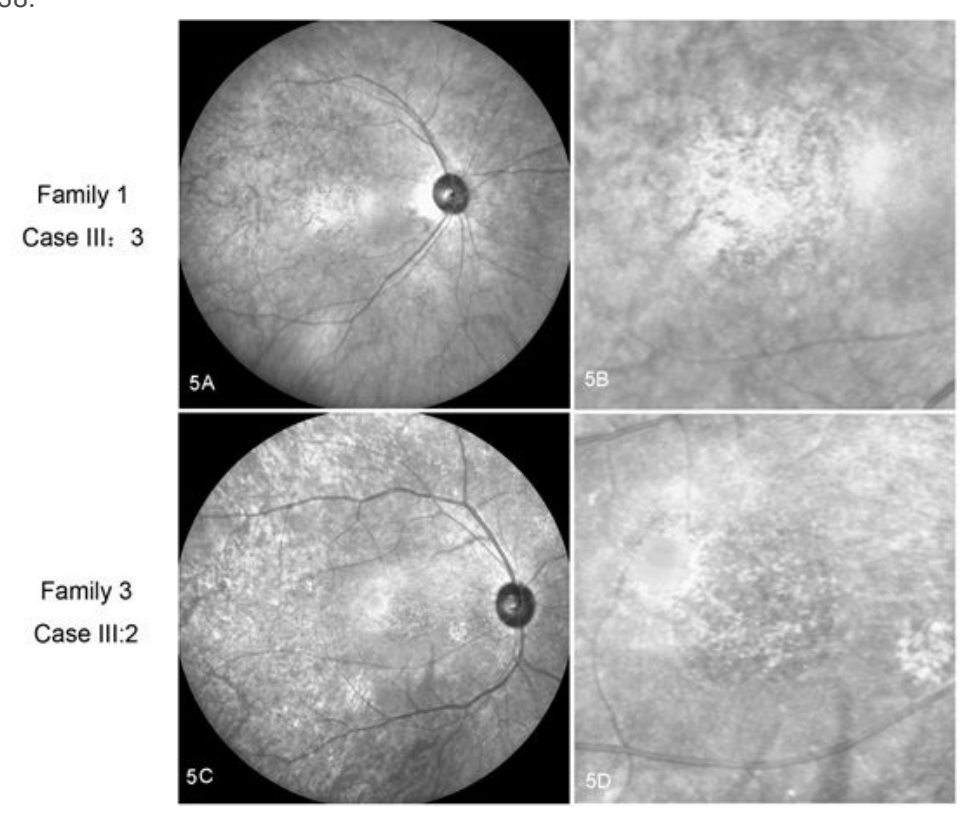


Figure 5

Photograph from the 633-nm laser of a scanning laser ophthalmoscope of Family 1-Case III:3 (a 54-year-old woman, right eye) (5A) and Family 3-Case III:2 (a 32 -year-old man, right eye) (5C). A coarse granular appearance was observed in both patients. Figures $5 \mathrm{~B}$ and $5 \mathrm{D}$ are the enlargement of Figures $5 \mathrm{~A}$ and $5 \mathrm{~B}$, respectively.

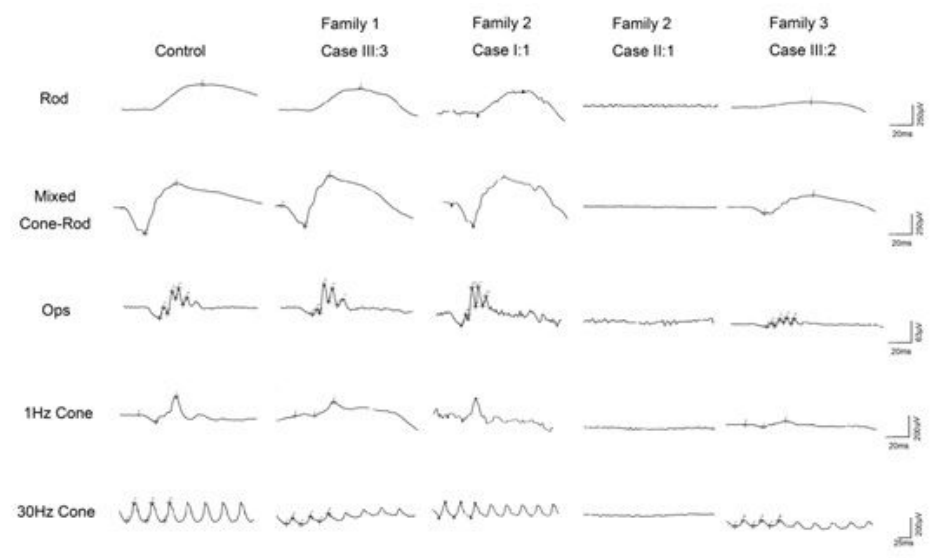

Figure 6

Standard ERGs in patients with AXTN7 variants. Representative rod, mixed cone-rod and cone ERGs from the patients compared with a normal subject. 